\title{
Future research in cancer survivorship
}

\author{
Raymond J. Chan ${ }^{1} \cdot$ Larissa Nekhlyudov $^{2}$. Saskia F.A. Duijts ${ }^{3}$. Shawna V. Hudson ${ }^{4}$. Jennifer M. Jones ${ }^{5}$. \\ Justin Keogh ${ }^{6} \cdot$ Brad Love $^{7} \cdot$ Maryam B. Lustberg $^{8} \cdot$ Anja Mehnert-Theuerkauf $^{9} \cdot$ Paul Nathan $^{10} \cdot$ Kirsten K. Ness $^{11}$.

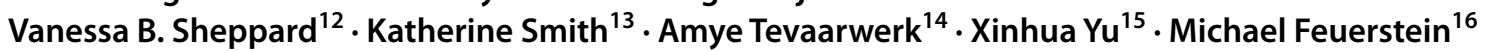

Accepted: 19 August 2021 / Published online: 30 August 2021

(c) The Author(s), under exclusive licence to Springer Science+Business Media, LLC, part of Springer Nature 2021

\section{Introduction}

Since the inception of the Journal of Cancer Survivorship (JCSU), the founding editor-in-chief, the associate editors, editorial board members, and peer reviewers have played important roles in influencing the knowledge base published in JCSU. The journal editors represent key disciplines, practice areas, and research expertise in cancer survivorship, which includes oncology and hematology, pediatric oncology, health psychology/behavior medicine, primary care, oncology nursing, exercise oncology, epidemiology, rehabilitation sciences, health services research, health policy, public health, and sociology. Over the years, the journal editors have worked to facilitate peer review and provide quality reviews to prospective authors. Earlier this year, Chan et al. [1] provided an overview of the evolution of JCSU since its inception in 2007 to 2020. Chan et al.'s [1] paper included a comprehensive description of the content, impact metrics, and top cited papers. In addition, they created a neural network using the key terms of published articles (Fig. 1) that depicted both minor and major themes published since the inception of JCSU.

As illustrated in Fig. 1, the most common theme was "quality of life." The neural network of key terms also indicated sub-themes including healthcare delivery and follow-up care; management of comorbidities and concurrent conditions; physical activity and exercise; healthy lifestyle/behaviors other than physical activity; disparity and social determinants of health; employment; management of symptom/toxicity/late effects; psycho-oncology/ psychosocial care, adolescent, and young adult (AYA) cancer survivorship; and health communications. This editorial provides our suggestions as journal editors on future evidence that can prove helpful in advancing knowledge, practice, and policy internationally. A summary of these research areas organized by the themes noted in the neural network of key terms can be found below and bulleted in Table 1.
Raymond J. Chan

Raymond.Chan@flinders.edu.au

1 Caring Futures Institute, College of Nursing and Health Sciences, Flinders University, Bedford Park, SA 5042, Australia

2 Brigham and Women's Hospital, Harvard Medical School, Boston, MA, USA

3 Netherlands Comprehensive Cancer Organisation (IKNL), Utrecht, Amsterdam University Medical Centers, Amsterdam, Netherlands

4 Rutgers Robert Wood Johnson Medical School and Rutgers Cancer Institute of New Jersey, New Brunswick, NJ, USA

5 Princess Margaret Cancer Centre, Toronto, Ontario, Canada

6 Bond University, Robina, Queensland, Australia

7 Center for Health Communication, University of Texas, Austin, TX, USA
8 MPH, Yale Cancer Center, New Haven, CT, USA

9 University Medical Center Leipzig, Leipzig, Germany

10 The Hospital for Sick Children, Toronto, ON, Canada

11 FAPTA, St. Jude Children's Research Hospital, Memphis, TN, USA

12 School of Medicine and the Massey Cancer Center Office of Outreach Engagement and Health Disparities, Virginia Commonwealth University, Richmond, VA, USA

13 Bloomberg School of Public Health, Johns Hopkins University, Baltimore, MD, USA

14 Carbone Cancer Center, University of Wisconsin School of Medicine and Public Health, Madison, WI, USA

15 University of Memphis School of Public Health, Memphis, TN, USA

16 Journal of Cancer Survivorship, Memphis, TN, USA 


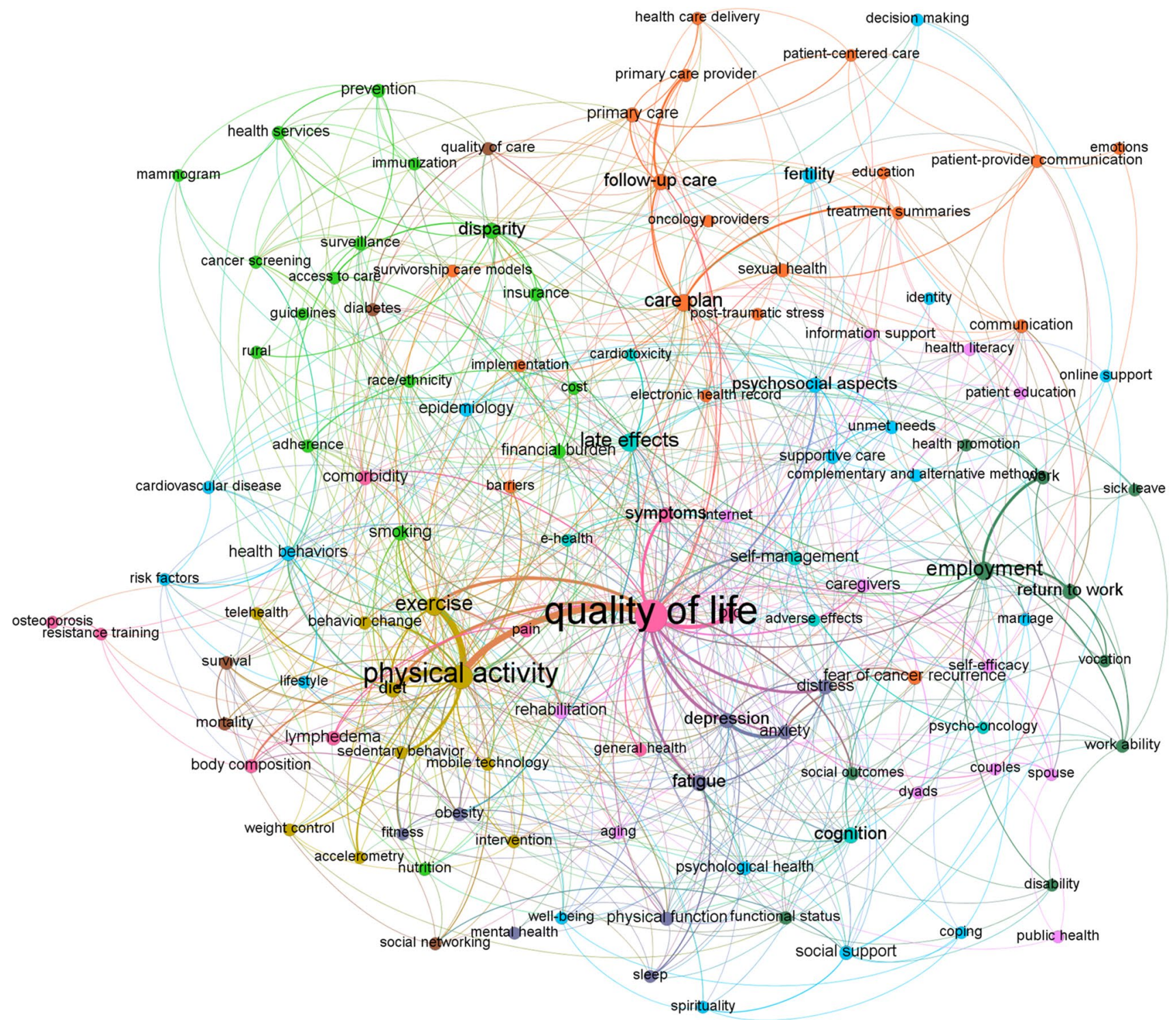

Fig. 1. Keyword co-occurrence networks for the Journal of Cancer Survivorship between 2007 and 2020 (reprinted with permission from: Chan RJ, Hollingdrake O, Bui U, Nekhlyudov L, Hart NH, Lui $\mathrm{C}-\mathrm{W}$, et al. Evolving landscape of cancer survivorship research: an analysis of the Journal of Cancer Survivorship, 2007-2020. J Cancer Surviv. 2021;15(4):651-8). Note. General areas represent topics of

\section{Healthcare delivery and follow-up care}

While published papers in the JCSU have focused on follow-up care and healthcare delivery for cancer survivors, there are topics that warrant further investigation. First, there is a need to examine tailored models of care that account for the fact that "one size is not likely to fit all" cancer survivors. It is important that type of care delivered, by whom, where, and for which survivors be expanded. This work should take into account the published papers as indicated in the neural network of all published papers in JCSU from 2007 to 2020. The research areas under each general topic are of particular interested to JCSU; however, as always, JCSU will consider any submission that falls within the journal's scope, as indicated on our website https://www.springer.com/journal/ 11764

following: age, risk for recurrence, subsequent malignancies, symptoms, supportive care needs, complex chronic conditions, and individual preferences. In testing models of care, it is important to acknowledge that survivorship care should be comprehensive, and not likely to be delivered by one type of healthcare provider. Interventions need to be targeted across different domains of care, engage specialists beyond oncology and primary care (e.g., rehabilitation specialists, cardiology), and include other staff integral in survivorship care delivery (e.g., various mental health specialists, nursing, financial navigators). The role 
Table 1. Research on cancer survivorship: areas for future considerations

Healthcare delivery and follow-up care

Future research should:

- Examine tailored models of care that account for risk of cancer recurrence, risk of secondary cancer, symptoms, supportive care needs and complex chronic conditions and emphasize patient self-management

- Establish interventions that are targeted across different domains of care, engage specialists beyond oncology and primary care, and include other staff integral in survivorship care delivery

- Examine the role of electronic health records and telehealth in healthcare delivery and measure their quality on both proximal and distal outcomes

- Incorporate the assessment and consideration of implementation science principles

- Establish interventions that are designed to promote sustainability, scalability, and adaptability

\section{Management of comorbidities and concurrent conditions}

Future research should:

- Focus on proactive communication and care-coordination, particularly on communication among survivors, primary care, and specialists

- Examine how electronic health records and other healthcare information technology can help and/or hinder communication and care coordination among survivors, primary care, and specialists across the cancer trajectory

- Evaluate global differences, with specific requests for the major continental hubs, including care in advanced and developing nations

Physical activity and exercise

Future research should:

- Focus on improving the translation of exercise oncology research into practice using a variety of quantitative and qualitative research designs

- Identify current translation approaches, outcomes, and ongoing challenges

- Examine oncologists' and other healthcare professionals' promotion of exercise/physical activity to their patients

- Investigate referral pathways to evidence-based exercise programs

- Identify cancer survivors' challenges in incorporating exercise/physical activity into their life

\section{Healthy lifestyle changes}

Future research should:

- Focus on establishing causal mechanisms between interventions and outcomes that are biologically plausible, clinically relevant, and meaningful to patients

- Go beyond a small observational study or non-randomized trial when there is already evidence to suggest that there is a relationship between an intervention approach and an important outcome

- Draw upon evidence-based theoretical models and behavior change techniques that have been shown to be effective at changing lifestyle behaviors

- Consider lifestyle interventions in both common and rarer cancers, as well as throughout the survivorship trajectory

- Measure long-term outcomes of interventions and maintenance of targeted behavior change after completion of structured programming

- Consider the use of mobile internet capable technologies and devices to promote behavior change

- Consider the efficacy of interventions in low and middle-income countries and in diverse populations

- Establish interventions that are that are cognizant of cultural context, feasibility, acceptability, and sustainability

Disparity and social determinants of health

Future research should:

- Examine practice, policy, innovative healthcare delivery models (e.g., multidisciplinary approaches, navigation), and/or community partnerships to address social determinants of health and/or health disparities among survivors

- Be conducted in partnership with diverse populations and investigate the underlying factors of social disparity

- Evaluate intervention approaches to improve disparate survivorship outcomes

- Examine intersectionality between race, geography, and gender identity

- Investigate disparities in health-related quality of life and financial toxicity

- Consider the context of other areas of survivorship such as health behaviors and communication

\section{Employment}

Future research should:

- Focus on the development of early interventions from diagnosis onwards, regarding return to work and continuation of work, to prevent the development of (financial) problems at later stages

- Focus on work issues in specific, vulnerable subgroups, particularly patients receiving advanced cancer care, those with less common tumor types, and those who experienced job loss or work disability

- Focus on improving the translation of "cancer and work" research into practice

\section{Management of symptoms/toxicity/late effects}

Future research should:

- Focus on longer-term follow-up for toxicity, function, and quality of life

- Investigate less evaluated toxicities such as financial, return to work, and fertility consequences over time

- Examine new toxicities and more unusual cancer symptoms as well as improving biological/mechanistic understanding of common toxicities

- Use real-world evidence to monitor outcomes in populations that are not typically included in trials 
Table 1. (continued)

\section{Psycho-oncology/psychosocial care}

Future research should:

- Establish psycho-oncological interventions for specific target groups, considering factors such as age, social condition, and comorbidities

- Focus on psychological comorbidities such as depression or fear of cancer recurrence and assess them in the long term

- Identify modifiable physical, behavioral, and social risk factors for psychosocial distress

- Focus on older survivors with severe physical impairments, and those in specific risk groups such as survivors in precarious economic circumstances

- Evaluate psycho-oncological interventions under everyday conditions in the sense of healthcare services research, and consider a plurality of therapy methods including mobile health devices

- Promote self-management skills and health literacy to improve quality of life in cancer survivors and family caregivers

\section{Adolescent and young adult care}

Future research should:

- Identify AYA responses to therapy and disease management strategies

- Identify optimal ways to provide adequate psychosocial support to AYA cancer survivors

- Establish developmentally appropriate multidisciplinary care that addresses fertility and sexuality, treatment adherence, potential for substance abuse/misuse, and support for physical changes associated with cancer and cancer care, while incorporating measurement and intervention rubrics that consider self-image, relationships, and both independent and shared decision-making

- Establish care that encourages peer support and that uses age-specific channels and communication mechanisms (e.g., social media)

- Explore the accelerated aging phenotype that is present in AYA populations post-cancer treatment

\section{Health communication}

Future research should:

- Develop opportunities to improve the impact of communication at every level from the individual-level clinical conversation between a patient and provider to small-group coaching

- Enhance the precision of message content and delivery in ways that persuasively influence attitudes and behavior(s) to improve a range of key areas, such as treatment adherence, behavioral interventions around exercise and nutrition, information exchange among specialists, emotional support resources, family interactions, peer connections, financial toxicity, uncertainty management, career disruption, and fertility concerns

- Develop effective systems to create two-way communication between survivors and providers such that survivor voices and input influence the clinical experience in substantial ways (monitor outcomes)

- Focus on improving consideration for how clinicians engage with people from different backgrounds and lived experiences

- Continue to advance digital technologies that present a range of options to improve and increasingly tailor communications targeted toward survivors

- Continue to maximize the quality and scope of data collection which can enable understanding of more nuances of people's journeys, ultimately improving resources available to support survivorship

of electronic health records and telehealth for delivering such tailored and comprehensive, yet equitable care should be further explored. Studies also need to determine clinical effectiveness in both proximal and distal outcomes over a longer temporal course as cancer survivors live longer. Information on cost and value using a range of economic evaluation approaches should be considered.

Future research should also evaluate self-management approaches in survivorship models of care, with providers working with survivors to identify supportive resources for financial navigation, symptom management, management of comorbidities, and care coordination. Lastly, there is a critical need for survivorship interventions and pragmatic trials that incorporate innovative assessment and implementation science. Interventions should be designed from the onset to facilitate sustainability, scalability, and adaptability when proven efficacious.

\section{Management of comorbidities and concurrent conditions}

As the population of people with cancer continues to age [2], the likelihood that they and their cancer care team will need to manage or co-manage concurrent medical conditions increases. While current literature acknowledges the importance of concurrent medical conditions, there has not been a sufficient focus on this area. Targeted interventions focusing on proactive communication and care coordination are needed. Real-world evidence regarding types and impact of communication strategies among survivors, primary care, and specialists following cancer diagnosis and treatment is required. As chronological aging places survivors at risk for age-related chronic medical conditions and long-term and late effects, there is a need to differentiate the impact of aging from the effects of the cancer and its treatment to 
better inform interventions. On the other hand, premature aging affecting those diagnosed at a younger age also needs further investigation and differentiation.

Future research should focus on examining how electronic health records and other healthcare information technologies can facilitate and/or hinder communication and care coordination among survivors, primary care, and other specialists across the cancer trajectory. Opportunities for "extending care beyond the oncology visit" to gather information about survivor well-being and status (e.g., electronic patient reported outcomes, at home monitoring, telemedicine) should be explored. The design, evaluation, and implementation of effective patient self-management approaches should incorporate clinicians, community professionals, and lay staff (neighborhood resources) and facilitate engagement with patient and caregiver stakeholders ensuring intervention relevance and acceptability.

\section{Physical activity and exercise}

Over the last three decades, considerable epidemiological and level-one evidence from systematic reviews of randomized controlled trials (RCT) has demonstrated widespread benefits of physical activity and exercise for optimal cancer survivorship. Specifically, this research indicates that a range of physical, psychosocial, and overall health benefits can be derived from exercise and persistent activity, with such benefits observed across a variety of types of exercise and activity, cancers, and at different points of the cancer survivorship journey [3]. As a result of this research, a variety of clinical practice guidelines for the incorporation of exercise/ physical activity into cancer care have been generated [4], although the majority of these guidelines are geared towards general cancer populations, and long-term maintenance of outcome is often not addressed.

While there is still the need for additional RCTs to determine the most effective exercise prescriptions across a multitude of cancer types, stages, and treatment options, perhaps the greatest requirement for future research in exercise oncology lies in the translation of "lab" findings into improved care practices. It has been proposed that oncologists and other healthcare professionals are ideally placed to promote exercise/physical activity and refer their patients (when required) to exercise professionals including exercise physiologists and physiotherapists [3]. Unfortunately, a recent systematic review [5] indicates that only a limited number of healthcare professionals routinely initiate discussions about exercise/physical activity with their patients, continuing to highlight persistent multiple barriers such as a lack of time, limited referral pathways, and a lack of perceived patient interest. Studies that focus on improving the translation of exercise oncology research into practice are needed. Such studies could use a variety of implementation and pragmatic designs involving quantitative and qualitative methods. The focus on topics might include the following: (1) oncologists' and healthcare professionals' effective promotion of exercise/physical activity to their patients; (2) referral pathways to evidence-based exercise and/or increased physical activity programs; and (3) cancer survivors' challenges and solutions to incorporating exercise/ physical activity into their everyday lives over the short and longer term. The evidence base for current translation approaches, ongoing challenges, solutions, and outcomes using rigorous study designs remain a priority.

\section{Healthy lifestyle interventions}

In terms of other healthy lifestyle changes, there is also a need for rigorous research on innovative approached to health behavior change that addresses cancer-related symptoms, a range of treatment side effects, and generally improves health status and quality of life among cancer survivors. There is still a need for well-designed studies to improve diet and sleep; reduce stress and improve mental health; build and maintain social networks and social support; support smoking cessation and reductions in alcohol use; and address challenges in intimate relationships.

Studies that focus on establishing causal mechanisms between interventions and outcomes that are clinically relevant and meaningful to patients should also be developed. Where there exists some indication of a relationship between an intervention approach and some important outcome, there is often a need for research that goes beyond small observational studies, or non-RCTs using and the use of convenience samples of survivors. Studies should draw upon evidencebased theoretical models and behavior change techniques that have shown not only to be effective at changing lifestyle behaviors-including evidence from outside of the cancer literature-but new approaches that might be specific to those living with a history of cancer and its treatment. There is a need for studies that consider lifestyle interventions in both common and more rare cancer types, as well as throughout survivorship.

Too few studies appropriately standardize intervention delivery, dosage, and duration; however, as is well known, studies that are taken seriously are cauterized as those with rigorous measurement of intervention delivery and study outcomes. There is also a need for interventions that are sensitive to cultural context, as well as feasibility, acceptability, and sustainability. There is a considerable need for studies that measure long-term outcomes of interventions and the maintenance of targeted behavior change after completion of structured programming or approaches that help maintain change. This is rarely examined in cancer survivors, limiting both the knowledge of long-term effectiveness and the translation into practice. Little is still known about 
the most effective behavior change techniques for promoting/maintaining positive long-term outcomes in survivors. Qualitative and mixed methods research designs can be useful for identifying reasons for persistent non-adherence and the impacts. Research should continue to consider the use of mobile internet capable technologies and devices to promote both short and long-term behavior change.

\section{Disparity and social determinants of health}

Despite advances in cancer treatment, it is recognized that disparities persist in cancer survivorship outcomes. Social determinants of health (factors which affect health beyond biology or genetic inheritance, such as employment, income, housing, nutrition and food insecurity, and transportation) are a major source of disparity and inequality in cancer care and outcomes [6]. Racial disparity across the continuum of cancer care is also well documented [7-9], with race and ethnicity remaining significant predictors of diagnosis, quality of health care received, treatment, and survival, even after socioeconomic conditions are accounted for [10]. There is need for future research on cancer survivorship to be conducted with diverse populations, and these studies should focus on the following: (1) investigating the underlying factors that explain health and social disparity (going beyond descriptions of well-documented disparities); (2) exploring new intervention approaches to improve disparate survivorship outcomes that are methodologically sound; and (3) identifying how to best capture and monitor these approaches.

Particular attention is necessary to prioritize research among survivors who identify in racial/ethnic as well as other minority groups. For example, African Americans face disproportionately higher adverse outcomes for many cancers compared to White Americans and even some other racial/ethnics groups $[9,11]$. Studies designed to minimize disparities in healthcare and health outcomes for these populations continue to inform us. Moreover, studies that advance understanding of the intersection of socioeconomic status, race, geography, gender, and sexual identity are critical to tackle disparities and promote equity in survivorship [12]. Disparities in health-related quality of life and financial toxicity are particularly relevant given the emphasis on valuebased care. It is recommended that evidenced-based strategies be prioritized to inform integration of cancer health equity practices by systematically addressing social determinants of health across the survivorship continuum.

Research regarding practice, policy, innovative healthcare delivery models (e.g., multidisciplinary approaches, navigation), and/or community partnerships to address social determinants of health and/or health disparities among survivors remain critical [6]. Social determinants of health and health disparities are cross-cutting and should be considered within the context of other areas of survivorship such as health behaviors, communication, and digital health advancement.

\section{Employment}

Globally, approximately $40-50 \%$ of all newly diagnosed cancer patients are of working age at time of diagnosis $[13,14]$. This percentage is expected to increase, with advances in cancer screening and treatment, along with the adjustment of the retirement age in some countries. About $64 \%$ of cancer survivors will return to work after their diagnosis $[15,16]$, and for those working at diagnosis (heterogeneous cancer types) about three quarters of long-term cancer survivors retain their working status over the long run [17]. Nevertheless, many cancer survivors experience long-term psychosocial (e.g., anxiety, loneliness), physical (e.g., fatigue, pain), cognitive (e.g., changes in ability to multitask, memory problems), and workplace challenges with co-workers or supervisors related to work [18-20]. Despite these difficulties, work-related interventions are often not provided to cancer survivors. One reason for this is only weak evidence from RCTs exists, with available evidence stating that multicomponent interventions marginally improve return to work in cancer survivors [21]. There is a need for additional work related RCTs across the various types of cancer survivors so that an evidence base can be generated to better inform practice specific to the concerns of cancer survivors with different types of cancers.

Numerous qualitative, longitudinal cohort, and registerbased studies have been conducted in the field of "cancer and work," identifying who returns to work [22]. While the development and evaluation of work-related interventions, to support these cancer survivors in their return to work and continuation of work, remains of utmost importance, future research in "cancer and work" needs to focus more specifically on early interventions, particularly on vulnerable subgroups of patients [23]. Timely attention to "work" may prevent financial problems, among others, at a later stage [24]. In addition, while numerous studies have addressed paid work problems in breast and colon cancer patients, research on work-related support for those who have experienced job loss or work disability, with less common tumor types and forms of work, is lacking (e.g., gig work).

Also, studies focusing on a range of innovative interventions including those that consider an effective range of workplace accommodations to mitigate challenges along with other types of support for survivors are needed. Improving outcomes related to return to work and maintenance of employment represent priorities. In addition, work concerns for specific subgroups, such as patients advanced cancer, are of interest to this journal. Finally, studies focusing on improving the translation of "cancer and work" research into everyday practice are also important. 


\section{Management of symptoms/toxicity/late effects}

Cancer survivors may live with the consequences of cancer and cancer therapies for many years, while clinicians caring for these survivors are faced with managing these consequences over an increasingly extended time frame. To address the needs of this growing population, evidencedbased management of symptoms, toxicities, and late or chronic effects is needed.

Over the last decade, diverse multidisciplinary teams have begun to address the gaps in symptom science and late effects. To continue making advances in this realm, additional efforts are needed to expand our knowledge base including studies that focus on the following: (1) longer-term follow-up to monitor and manage toxicities and relevant outcomes related to quality of life; (2) evaluation of less investigated toxicities such as financial and fertility consequences over time; (3) identifying and understanding new toxicities and more unusual symptoms as well as improving biologic and mechanistic understanding of common toxicities; and (4) using real-world evidence to monitor outcomes in populations that are not likely to be included in trials (e.g., older populations, those with locally recurrent or multiple primary tumors).

\section{Psycho-oncology/psychosocial care}

In recent decades, psycho-oncology research has provided profound and differentiated evidence on the spectrum of psychosocial stress and has revealed prevalence and risk factors for psychological distress and mental disorders that influence morbidity and even mortality in cancer survivors $[25,26]$. There is a need for epidemiological studies on the long-term assessment of psychological comorbidities such as depression or fear of cancer recurrence. Along with psychological comorbidities, modifiable physical, behavioral, and social risk factors for psychosocial distress should be identified. Greater attention should be paid to the growing group of older survivors and especially those with a high degree of frailty, severe physical impairments, and to specific risk groups such as survivors in precarious economic circumstances. Family caregivers should be considered a target population of interest, as their psychosocial burdens are considerable and, at the same time, they play a significant role in the process of survivors' coping, adaptation to the disease, and self-management. The study of psychosocial resources and factors that contribute to the resilience of patients and family caregivers should be emphasized. Survivorship research should also focus on methodologically robust interventions for specific target groups, sensitive to factors such as age, social conditions, and comorbidities. It is also important to evaluate psycho-oncological interventions under "everyday" conditions using a range of pragmatic evaluative designs. There is also a need to consider the appropriateness of applying flexible delivery of psychotherapy methods including individual, couple, and group interventions or interventions via mobile health devices. In addition to the reduction of psychological distress, the promotion of self-management skills and health literacy is of great importance to improve quality of life in cancer survivors and family caregivers [27].

\section{Adolescent and young adults}

Adolescents and young adults (AYA) diagnosed with cancer face unique challenges compared to individuals diagnosed as children or older adults, both during cancer therapy and survivorship. Continued multidisciplinary research focused on AYA-specific care models is necessary to address the immediate needs of this population and to provide evidence to guide medical and psychosocial care that optimizes longterm outcomes. In addition to clinical trials focused on responses to therapy and disease management (that include or are designed specifically for AYAs with cancer or a history of cancer and its treatment), multidisciplinary interventions should address fertility and sexuality, treatment adherence, potential for substance abuse/misuse, and support for physical changes associated with cancer and cancer care, while incorporating measurement and intervention rubrics that consider self-image, relationships, and both independent and shared decision-making. Care that encourages peer support and that uses age-specific channels and communication mechanisms (e.g., social media) is important because doing so meets these people where they are most comfortable, instead of where healthcare professionals want them to be. After therapy is complete, AYA survivors may require educational support, career counseling, and help locating financial resources as they recover and deal with long-term effects from their experiences.

Like childhood cancer survivors, AYA survivors are at risk for chronic health conditions and mortality at an earlier age than peers. Transitions to regular medical care may be difficult for AYAs after cancer therapy, particularly because many primary care providers do not understand the unique needs of AYAs and are often uncomfortable taking on their medical care. Accelerated aging in this population may look to a provider like poor fitness rather than treatmentrelated lack of physiologic reserve. This phenotype may be responsive to specific interventions that address diminished response to usual lifestyle recommendations such as diet and exercise. Thus, there is a need to increase the general medical community's awareness of the possibility of this post-cancer care phenotype. Additionally, although the Children's Oncology Group (COG) has published guidelines for medical screening of survivors, few providers are aware of this resource. The length of the COG recommendations may 
seem unwieldy, so simplification is ongoing internationally. Unfortunately, these guidelines do not yet address specific screening to identify psychosocial problems among survivors. Critical work is also needed to identify the best ways to provide adequate psychosocial support to AYA cancer survivors.

\section{Health communication}

Improved health communication provides a major opportunity and area of focus in the future of survivorship, as evidence-based information can improve the lived experience of these survivors. There is opportunity to improve the impact of communication from individual-level clinical conversations between a patient and provider to small-group coaching (e.g., use in dietetic support to media reminders about keeping up with regular scans). Each aspect of survivorship would benefit from increased health communication research to enhance the precision of message content and delivery in ways that persuasively influence attitudes and behaviors to improve a range of key areas, such as treatment adherence, behavioral interventions around exercise and nutrition, information exchange among specialists, emotional support resources, family interactions, peer connections, financial toxicity, uncertainty management, career disruption, and fertility concerns.

Additionally, more research is needed on effective systems to create two-way communication among survivors, their families/caregivers, and providers. Such approaches will enhance survivor voices and input that will influence the clinical experience in substantial ways and reduce latepresenting effects around psychosocial issues and life-course disruption. It is also important to consider how clinicians engage with people from different backgrounds and lived experiences. Another area of future research in communication is the use of digital technologies which continue to present a range of options to improve and increasingly tailor communications targeted toward different types of cancer survivors. This also may help improve the quality and scope of data that can be collected to understand more nuances of people's journeys and ultimately improve resources available to support survivorship.

\section{Conclusion}

The editors of this journal highlighted some research questions or gaps in knowledge organized using the neural network analysis of actual studies published in JCSU over the years as a framework. Each section has focused on the editors view of areas for new knowledge within major themes based on a collective experience of editorial, clini$\mathrm{cal}$, and research perspectives. An important message of all suggestion for future research, perhaps not unexpectedly, is that this research should use rigorous methodology and provide innovative information. Remember, the purpose of this journal is to help move the knowledge base of cancer survivorship.

Lastly, as in many areas of cancer research, there remains a need for evaluation of the efficacy of interventions in low and middle-income countries and diverse populations. Multi-national, trans-disciplinary research incorporating expertise from implementation science and technology is seen as increasingly necessary to provide innovative care solutions to the complex problems facing many cancer survivors. We believe that the suggestions presented in this editorial can help provide a framework for future studies that further our understanding of cancer survivorship and improve public health, quality care, and quality of life in this global population.

Acknowledgement The authors thank Dr Michelle Mollica for her contribution towards and critical review of the manuscript.

Funding Prof Raymond Chan receives salary support from the National Health and Medical Research Council (APP1194051).

\section{References}

1. Chan RJ, Hollingdrake O, Bui U, Nekhlyudov L, Hart NH, Lui $\mathrm{C}-\mathrm{W}$, et al. Evolving landscape of cancer survivorship research: an analysis of the Journal of Cancer Survivorship, 2007-2020. J Cancer Surviv. 2021;15(4):651-8. https://doi.org/10.1007/ s11764-021-01042-6.

2. Bluethmann SM, Mariotto AB, Rowland JH. Anticipating the "silver tsunami": prevalence trajectories and comorbidity burden among older cancer survivors in the United States. Cancer Epidemiol Biomarkers Prev. 2016;25(7):1029-36. https://doi.org/10. 1158/1055-9965.EPI-16-0133.

3. Brown JC, Ligibel JA. Putting exercise into oncology practice: State-of-the-science, innovation, and future directions. Cancer J. 2019;25(5):316-9. https://doi.org/10.1097/ppo.0000000000 000397.

4. Shallwani SM, King J, Thomas R, Thevenot O, De Angelis G, Aburub AS, et al. Methodological quality of clinical practice guidelines with physical activity recommendations for people diagnosed with cancer: a systematic critical appraisal using the AGREE II tool. PLoS One. 2019;14(4):e0214846. https://doi.org/ 10.1371/journal.pone.0214846.

5. Alderman G, Semple S, Cesnik R, Toohey K. Health care professionals' knowledge and attitudes toward physical activity in cancer patients: a systematic review. Semin Oncol Nurs. 2020;36(5):151070. https://doi.org/10.1016/j.soncn.2020.151070.

6. Alcaraz KI, Wiedt TL, Daniels EC, Yabroff KR, Guerra CE, Wender RC. Understanding and addressing social determinants to advance cancer health equity in the United States: A blueprint for practice, research, and policy. CA Cancer J Clin. 2020;70(1):3146. https://doi.org/10.3322/caac.21586.

7. Zhang C, Zhang C, Wang Q, Li Z, Lin J, Wang H. Differences in stage of cancer at diagnosis, treatment, and survival by race and ethnicity among leading cancer types. JAMA Netw Open. 
2020;3(4):e202950. https://doi.org/10.1001/jamanetworkopen. 2020.2950

8. Ellis L, Canchola AJ, Spiegel D, Ladabaum U, Haile R, Gomez SL. Racial and ethnic disparities in cancer survival: the contribution of tumor, sociodemographic, institutional, and neighborhood characteristics. J Clin Oncol. 2018;36(1):25-33. https://doi.org/ 10.1200/jco.2017.74.2049.

9. DeSantis CE, Miller KD, Goding Sauer A, Jemal A, Siegel RL. Cancer statistics for African Americans, 2019. CA Cancer J Clin. 2019;69(3):211-33. https://doi.org/10.3322/caac.21555.

10. Institute of Medicine (US) Committee on understanding and eliminating racial and ethnic disparities in health care. In: Smedley BD, Stith AY, Nelson AR, editors. Unequal Treatment: Confronting Racial and Ethnic Disparities in Health Care. Washington (DC): National Academies Press (US); 2003.

11. Tucker-Seeley RD. Social determinants of health and disparities in cancer care for Black people in the United States. JCO Oncol Pract. 2021;17(5):261-3. https://doi.org/10.1200/op.21.00229.

12. O'Malley D, Tsui J, Davis S, Hudson S. Disparities. In: Feuerstein M, Nekhlyudov L, editors. Handbook of cancer survivorship. New York: Springer Science+Business Media; 2018. p. 73-89.

13. de Boer AG. The European Cancer and Work Network: CANWON. J Occup Rehabil. 2014;24(3):393-8. https://doi.org/10. 1007/s10926-013-9474-5.

14. Ferlay J, Steliarova-Foucher E, Lortet-Tieulent J, Rosso S, Coebergh JW, Comber H, et al. Cancer incidence and mortality patterns in Europe: estimates for 40 countries in 2012. Eur J Cancer. 2013;49(6):1374-403. https://doi.org/10.1016/j.ejca.2012.12.027.

15. Feuerstein M, Todd BL, Moskowitz MC, Bruns GL, Stoler MR, Nassif T, et al. Work in cancer survivors: a model for practice and research. J Cancer Surviv. 2010;4(4):415-37. https://doi.org/10. 1007/s11764-010-0154-6.

16. Mehnert A. Employment and work-related issues in cancer survivors. Crit Rev Oncol Hematol. 2011;77(2):109-30. https://doi. org/10.1016/j.critrevonc.2010.01.004.

17. de Boer AG, Torp S, Popa A, Horsboel T, Zadnik V, Rottenberg $\mathrm{Y}$, et al. Long-term work retention after treatment for cancer: a systematic review and meta-analysis. J Cancer Surviv. 2020;14(2):135-50. https://doi.org/10.1007/s11764-020-00862-2.

18. Duijts SFA, van Egmond MP, Spelten E, van Muijen P, Anema JR, van der Beek AJ. Physical and psychosocial problems in cancer survivors beyond return to work: a systematic review. Psychooncology. 2014;23(5):481-92. https://doi.org/10.1002/pon.3467.
19. Moskowitz MC, Todd BL, Chen R, Feuerstein M. Function and friction at work: a multidimensional analysis of work outcomes in cancer survivors. J Cancer Surviv. 2014;8(2):173-82. https:// doi.org/10.1007/s11764-013-0340-4.

20. Zegers AD, Coenen P, van Belzen M, Engelen V, Richel C, Dona DJS, et al. Cancer survivors' experiences with conversations about work-related issues in the hospital setting. Psychooncology. 2021;30(1):27-34. https://doi.org/10.1002/pon.5529.

21. de Boer AG, Taskila T, Tamminga SJ, Frings-Dresen MH, Feuerstein $\mathrm{M}$, Verbeek JH. Interventions to enhance return-to-work for cancer patients. Cochrane Database Syst Rev. 2011;2:Cd007569. https://doi.org/10.1002/14651858.CD007569.pub2.

22. Klaver KM, Duijts SFA, Engelhardt EG, Geusgens CAV, Aarts MJB, Ponds RWHM, et al. Cancer-related cognitive problems at work: experiences of survivors and professionals. J Cancer Surviv. 2020;14(2):168-78. https://doi.org/10.1007/s11764-019-00830-5.

23. Chan RJ, Cooper B, Gordon L, Hart N, Tan CJ, Koczwara B, et al. Distinct employment interference profiles in patients with breast cancer prior to and for 12 months following surgery. BMC Cancer. 2021;21(1):883. https://doi.org/10.1186/s12885-021-08583-0.

24. Chan RJ, Gordon L, Zafar SY, Miaskowski C. Financial toxicity and symptom burden: what is the big deal? Support Care Cancer. 2018;26(5):1357-9. https://doi.org/10.1007/s00520-018-4092-6.

25. Kuhnt S, Brähler E, Faller H, Härter M, Keller M, Schulz H, et al. Twelve-month and lifetime prevalence of mental disorders in cancer patients. Psychother Psychosom. 2016;85(5):289-96. https://doi.org/10.1159/000446991.

26. Hartung TJ, Brähler E, Faller H, Härter M, Hinz A, Johansen C, et al. The risk of being depressed is significantly higher in cancer patients than in the general population: prevalence and severity of depressive symptoms across major cancer types. Eur J Cancer. 2017;72:46-53. https://doi.org/10.1016/j.ejca.2016.11.017.

27. Howell D, Mayer DK, Fielding R, Eicher M, Verdonck-de Leeuw IM, Johansen C, et al. Management of cancer and health after the clinic visit: a call to action for self-management in cancer care. J Natl Cancer Inst. 2021;113(5):523-31. https://doi.org/10.1093/ jnci/djaa083.

Publisher's note Springer Nature remains neutral with regard to jurisdictional claims in published maps and institutional affiliations. 